\title{
Real-time quantum calculations of phase shifts using wave packet time delays
}

\author{
Erik Gustafson $\odot,{ }^{1}$ Yingyue Zhu, ${ }^{2}$ Patrick Dreher® ${ }^{3,4}$ Norbert M. Linke, ${ }^{2}$ and Yannick Meurice ${ }^{1}$ \\ ${ }^{1}$ Department of Physics and Astronomy, The University of Iowa, Iowa City, Iowa 52242, USA \\ ${ }^{2}$ Joint Quantum Institute and Department of Physics, University of Maryland, \\ College Park, Maryland 20740, USA \\ ${ }^{3}$ Department of Electrical and Computer Engineering, North Carolina State University, \\ Raleigh, North Carolina 27695, USA \\ ${ }^{4}$ Department of Physics, North Carolina State University, Raleigh, North Carolina 27695, USA
}

(Received 25 April 2021; accepted 23 August 2021; published 16 September 2021)

\begin{abstract}
We present a method to extract the phase shift of a scattering process using the real-time evolution in the early and intermediate stages of the collision in order to estimate the time delay of a wave packet. This procedure is convenient when using noisy quantum computers for which the asymptotic out-state behavior is unreachable. We demonstrate that the challenging Fourier transforms involved in the state preparation and measurements can be implemented in $1+1$ dimensions with current trapped ion devices and IBM quantum computers. We compare quantum computations of the time delays obtained in the one-particle quantum mechanics limit and the scalable quantum field theory formulation with accurate numerical results. We discuss the finite volume effects in the Wigner formula connecting time delays to phase shifts. The results reported involve two- and four-qubit calculations, and we discuss the possibility of larger scale computations in the near future.
\end{abstract}

DOI: 10.1103/PhysRevD.104.054507

\section{INTRODUCTION}

In recent years, the idea of simulating quantum field theory with quantum computers has gained considerable interest [1]. In the context of high-energy and nuclear physics, a long term motivation is to develop quantum computing methods that perform real-time evolution for lattice quantum chromodynamics (QCD). This is an $a b$-initio, ultraviolet complete, theory of strong interactions which has been very successful in describing the static properties of hadrons and nuclei [2]. Importance sampling methods, used successfully today for lattice QCD at Euclidean time, are not effective at dealing with the rapid oscillations of real-time unitary operators acting on large Hilbert spaces. Currently, physicists resort to non-ab-initio algorithms such as Pythia and Herwig [3,4] to interpret hadronic collider data. Doing ab-initio calculations for jet physics would represent a major accomplishment and is a long-term goal for the high-energy community. Strategies to deal with the related question of parton distributions are outlined in Refs. [5,6]. Progress for out-of-equilibrium

Published by the American Physical Society under the terms of the Creative Commons Attribution 4.0 International license. Further distribution of this work must maintain attribution to the author(s) and the published article's title, journal citation, and DOI. Funded by SCOAP ${ }^{3}$. processes in many-electron systems and information paradoxes in quantum gravity [7] would also have a large potential impact.

As quantum computers offer new approaches to various sign problems, it is common to advocate following the sequence of models that has been successful for the development of lattice QCD at Euclidean time on classical computers $[8,9]$. The first step in this sequence is to study the quantum Ising model (QIM) on today's noisy intermediate-scale quantum (NISQ) hardware. Real time evolution involving a limited number of sites for the QIM has already been attempted using a few qubits on gate based quantum computers [10-23], as well as developments in progress for more complicated models [24-38]. For processes involving a few Trotter steps, errormitigation methods such as zero-point extrapolation [39], written for a generic noise that can be intentionally increased in order to attempt an extrapolation to zero noise, have been applied successfully [28,40,41]. It has been shown [13] that by modeling four qubits on an IBM Q quantum computing hardware platform these mitigation methods together with using significantly larger Trotter steps $[12,42,43]$ provide a reasonable extrapolation for times of the order of the approximate periodicity of the problems considered.

We will demonstrate that these recently developed methods can be used for state-of-the-art NISQ devices to 


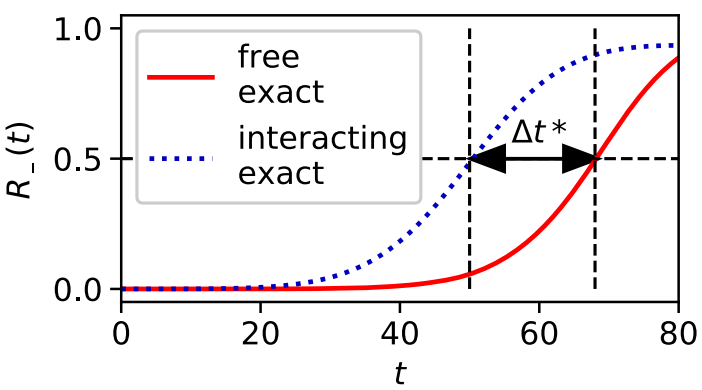

FIG. 1. Illustration of the measurement of the time delay between the free and interacting wave packets. The normalized reflection probability $R_{-}(t)$ is defined in Eq. (9).

prepare and evolve suitable wave packets for the QIM. In order to optimize the use of limited resources, we will focus on the reduced problem of a particle coming from the left, rebounding on a wall and returning to the left. We show that it is possible to project the wave-function in the early stages of a collision process onto momentum states and to pinpoint a time $t^{\star}$ that corresponds to the middle of the collision with the wall. This time can be estimated by computing the time when the probability for the approximate momentum of the initial wave packet and its opposite are equal. In practice, this can be done by introducing a normalized probability $R_{-}(t)$ for the reflection, defined later in Eq. (9) and which takes the value 0.5 at $t^{\star}$. By introducing an extra interaction close to the wall, we obtain a time delay $\Delta t^{\star}$ illustrated in Fig. 1 . We will show that $\Delta t^{\star}$ is half of the time delay $\Delta t_{W}$ invoked in Wigner formula [44], provided in Eq. (6), to estimate the derivative of the phase shift with respect to the momentum.

Phase shifts are a key measurement in the scattering process and represent the total change of phase due to interactions. Significant progress has been made in calculating them from lattice QCD in Euclidean time [45-50]. In standard textbooks, phase shifts and scattering amplitudes are estimated from asymptotic data long after the collision processes have occurred. However, for NISQ devices with limited coherence time or gate-depth, using the information from the early stages of the collision is advantageous. We show that this idea can be implemented on both a quantum computer using superconducting transmon qubits and a trapped ion system operating at the University of Maryland [51].

The article is organized as follows. We introduce the quantum Ising model with an extra interaction and its Hilbert space. We show that it is possible to implement the three steps of the calculation of the S-matrix elements: (1) preparation of the initial state, (2) real-time evolution, and (3) measurement of the probability for a particular final state. We then extract the phase shift by comparing the cases with and without an external potential. Steps (1) and (3) involve Fourier transforms and are very challenging with NISQ devices. This is why we first restrict ourselves to the quantum mechanics limit where one-particle states interact with an external potential localized at one site. We then show that it is possible to extend the computations to the case of the quantum field theory formulations [12] that require more qubits but are guaranteed to scale efficiently for larger volume. More specifically, it can shown [52] that for finite range interactions, for instance involving only nearest neighbor degrees of freedom, the computing timescales like the size of the system.

\section{MODEL CALCULATIONS OF PHASE SHIFTS}

We consider the transverse-field Ising model in one spatial dimension,

$$
\hat{H}_{0}=-J \sum_{i=1}^{N-1} \hat{\sigma}_{i}^{x} \hat{\sigma}_{i+1}^{x}-h_{T} \sum_{i=1}^{N} \hat{\sigma}_{i}^{z}
$$

The on-site energy $h_{T}$ is often called a transverse magnetic field and the ferromagnetic nearest-neighbor interaction $J$ is responsible for particle hopping, creation and annihilation. This model is very well understood [9] and discussed for NISQ devices [10-13]. It is equivalent to a theory of free fermions with subtle effects from the boundary.

In order to perform Fourier transforms with a reduced number of qubits, we first consider the quantum mechanics limit $J \ll h_{T}$, where the model consists of energy bands that can be assigned a particle number. This amounts to neglecting terms of the form $\hat{\sigma}_{i}^{+} \hat{\sigma}_{i+1}^{+}$and their conjugates. This reduces the size of the Hilbert space from $2^{N}$ to $N$ and also and analytic calculations [12] are possible in this approximation.

For problems invariant under translations and rotations, the standard quantum mechanics two-particle-scattering problem can be reduced to a radial Schroedinger equation in an effective potential. In one spatial dimension, the simplest case of effective potential for the reduced problem is a step adjacent to an infinite wall. To be concrete, we can think of a particle coming from minus infinity and moving toward a wall. As it approaches the wall, it reaches a potential step which is defined as positive and constant. The phase shift due to this type of potential is a standard textbook problem.

In the Ising model, nontrivial interactions capable of generating a phase shift can be introduced with an extra term

$$
\hat{H}_{\mathrm{int}}=U \sum_{i=1}^{N-1} \hat{\sigma}_{i}^{z} \hat{\sigma}_{i+1}^{z} .
$$

For the reduced one-particle problem with a right wall, the interaction of Eq. (2) introduces an effective potential at the end of the chain. This can be seen in the one-particle basis. If the particle is away from the wall and has a neighbor on 
each site, it creates an energy $-2 U$, but if it next to the wall, it only creates an energy $-U$ which is $+U$ above the energy when it is away.

This discrete Schrödinger equation obtained in the limit of small $J$, with $U=0$, and no boundaries admits plane wave solutions $e^{ \pm i k x}$ with energy

$$
E(k)=2 J(1-\cos (k)) .
$$

An additive constant has been adjusted in order to have zero energy at zero momentum. If we introduce a right wall and impose $\psi(N+1)=0$, we need a relative phase $-e^{i 2 k(N+1)}$ for the reflected wave $e^{-i k x}$. If, in addition, we also introduce a repulsive potential $U>0$ at the rightmost site $N$, the problem with a right wall can be solved by introducing the phase shift. A short calculation shows that:

$$
e^{i 2 \delta(k)}=e^{-i 2 k} \frac{U+J e^{i k}}{U+J e^{-i k}} .
$$

Notice that this formula is independent of $N$, because so far there is no left wall. In practice, such left wall will be introduced to restrict the problem to $N$ sites. By imposing $\psi(0)=0$, we obtain a Luscher formula

$$
\delta(k)=-k(N+1) \quad \bmod \pi,
$$

which introduces a restriction on the momenta. The restriction to a finite number of sites implies a $N$-dependence that we will discuss below.

The time delay $\Delta t_{W}$ of a wave packet with a sharply defined momentum $k$ is related to the derivative of the phase shift by the Wigner formula [44]:

$$
\Delta t_{W}=2 \delta^{\prime}(k) /(\partial E / \partial k),
$$

where $\partial E / \partial k$ is the group velocity, which in our case is $2 J \sin (k)$. This formula is obtained by considering the superposition of two plane waves with infinitesimally close momenta and energies and calculating the time difference for the two waves to be in phase at a given location with and without interaction. This comparison needs to be done at a time sufficiently longer than the time when the interaction takes place. From Eq. (4) we get:

$\Delta t_{W}=\left(-1+J \frac{U \cos (k)+J}{U^{2}+J^{2}+2 J U \cos (k)}\right) / J \sin (k)$.

The time delay $\Delta t_{W}$ can also be estimated from the first half of the real-time evolution of the scattering process and that it is actually twice the time delay $\Delta t^{\star}$ already mentioned in Fig. 1. For this purpose, we define the probabilities to be in the $| \pm k\rangle$ state

$$
P_{ \pm}(t) \equiv|\langle \pm k \mid \psi(t)\rangle|^{2}
$$

and their normalized versions

$$
R_{ \pm}(t) \equiv \frac{P_{ \pm}(t)}{P_{+}(t)+P_{-}(t)}
$$

which by design satisfy

$$
R_{+}+R_{-}=1 .
$$

With this normalization $R_{+}(t)$ and $R_{-}(t)$ get interchanged under time-reversal with respect to $t^{\star}$. The real-time evolution provides the time $t^{\star}$ necessary to reach the symmetric situation where $P_{+}\left(t^{\star}\right)=P_{-}\left(t^{\star}\right)$ and $R_{-}\left(t^{\star}\right)=0.5$. This corresponds to the time where a classical particle would hit the wall. We can then compare $t^{\star}$ in the case where $U=0$ and a nonzero value. We call these times $t_{\text {free }}^{\star}$ and $t_{\text {int }}^{\star}$ respectively. We define the difference

$$
\Delta t^{\star} \equiv t_{\mathrm{int}}^{\star}-t_{\text {free }}^{\star}
$$

and will argue that

$$
\Delta t^{\star}=\frac{\Delta t_{W}}{2}
$$

This relation can be justified from the time-reversal argument that after $t_{\text {int }}^{\star}$ only half of the phase shift, $\delta(k)$, has built up while the other half builds after $t^{\star}$. This is actually why historically, the total phase shift is denoted $2 \delta(k)$.

In order to confirm this statement, we made a numerical calculation with a number of sites $N=128$. This number is larger than the number that we will use later $(N=4)$ and allows us to minimize the finite volume effects. The results are shown in Fig. 2 and support Eq. (11).

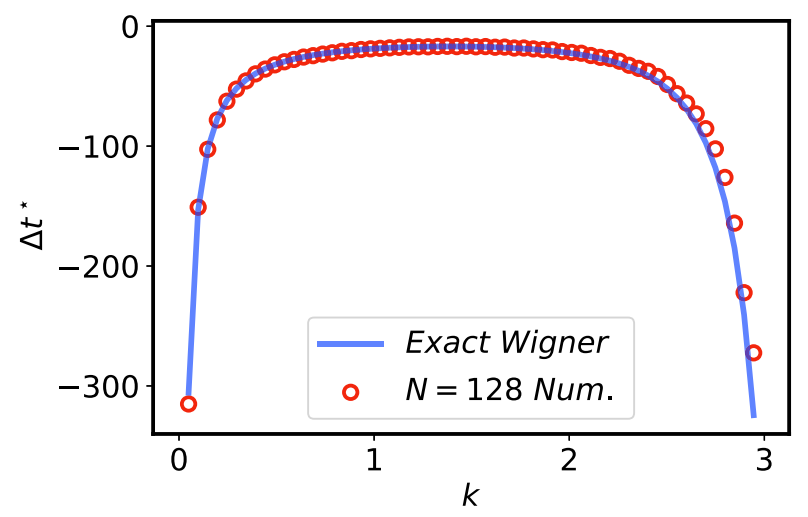

FIG. 2. Comparison of numerical $\Delta t^{*}$ with the analytical formula Eq. (7) for $N=128$. 
We now briefly explain how we calculated $\Delta t^{*}$. First, it is important to prepare a wave packet that is broad enough in space to have sharply defined momentum but not too broad so that it does not reflect too early. In the numerical calculations, we used an initial wave packet which in lattice units reads

$$
\psi(x) \propto e^{i k x-(x-N / 2)^{2} /(N / 4)^{2}} .
$$

Second, we used a sigmoid parametrization for the normalized reflection probability

$$
R_{-}(t) \simeq 1 /\left(1+\exp \left(-\left(\frac{t-t^{\star}}{w}\right)\right)\right) \text {. }
$$

Empirically, this provides very good fits of the numerical data. The parameter $w$ describes the width of the transition region in time units and $t^{\star}$ the time such that $R_{-}\left(t^{\star}\right)=0.5$ exactly. The quality of the sigmoid fit is illustrated in Fig. 3. The fit was performed by calculating the continuous time evolution by exact diagonalization and doing a sigmoid fit with six points in time, as we will do in the next section. It is useful to notice that the timescale for the duration of the collision is roughly proportional to $N$. In order to get an idea how the phase shifts change when we decrease $N$, we plotted $\delta(k)^{\prime}$ for $N=32,64$ and 128 in Fig. 4. This illustrates the magnitude of the volume effects which are clearly more pronounced when $k$ increases. Note that some integration procedure is needed in order to extract $\delta(k)$. From Eq. (4), we can expand $\delta(k)$ in power of $k$. For our numerical values $J=0.02$ and $U=0.03$, we obtain

$$
\delta(k) \simeq-0.6 k+0.008 k^{3}+\ldots
$$

For $0 \leq k \leq 0.5$ the correction to the linear approximation is less then one percent, justifying the approximation

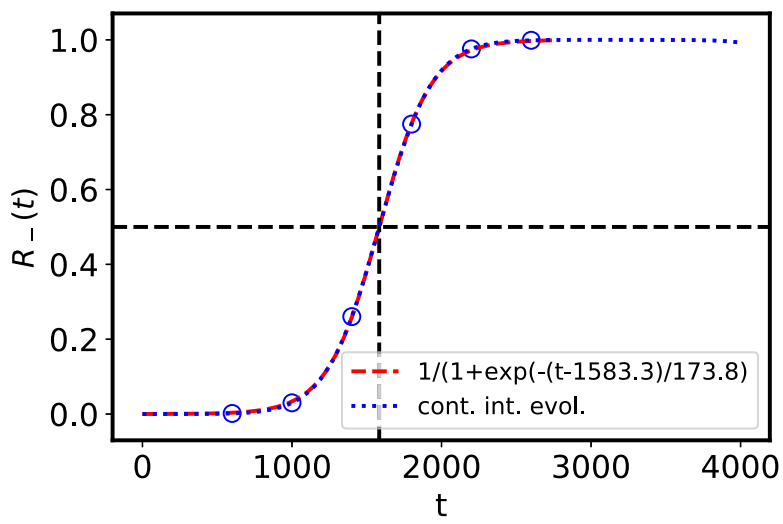

FIG. 3. Comparison of the continuous evolution of $R_{-}(t)$ and a sigmoid fit at six equally spaced times. The fitted values are $t^{\star}=$ 1583.3 and $w=173.8$.

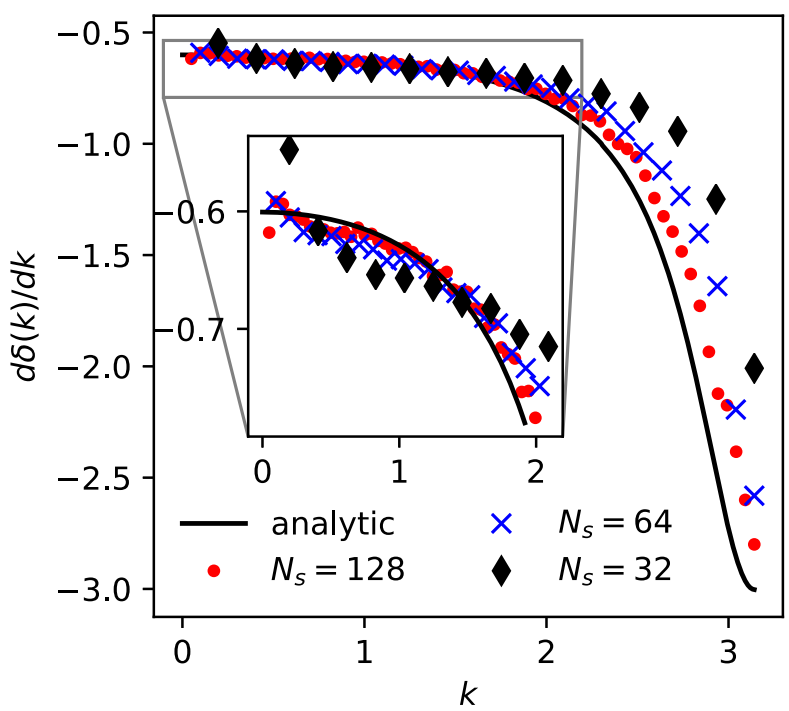

FIG. 4. Derivative of the phase shift with respect to momentum calculated numerically using the time delays and Eq. (6) for $N=32,64$ and 128 compared to the infinite volume results rom Eq. (7).

$$
\delta(k) \simeq \delta^{\prime}(0) k .
$$

This can be used to initialize the integration process to extract $\delta(k)$ from $\delta^{\prime}(k)$ obtained from the time delay.

\section{III. $N=4$}

Specializing to the case $N=4$, we have, up to an unimportant additive constant, the following effective Hamiltonian matrix:

$$
\hat{H}_{\mathrm{eff}}=\left(\begin{array}{cccc}
0 & -J & 0 & 0 \\
-J & 0 & -J & 0 \\
0 & -J & 0 & -J \\
0 & 0 & -J & U
\end{array}\right) .
$$

This allows us to reduce the Hilbert space from four qubits needed for the $N=4$ field theory problem to two qubits for the one particle limit. The remapping is shown in Eq. (18) with the correspondence illustrated in Fig. 5:

$$
\begin{aligned}
|1000\rangle & \rightarrow|00\rangle, & |0100\rangle & \rightarrow|01\rangle \\
|0010\rangle & \rightarrow|10\rangle, & |0001\rangle & \rightarrow|11\rangle .
\end{aligned}
$$

The Hamiltonian in Eq. (17) can now be written as 


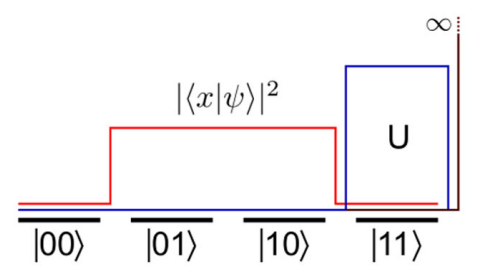

FIG. 5. Visual depiction of the qubit states (black dashes), potential for the interacting (blue) and noninteracting case (dark red), and initial wave packet (light red).

$$
\begin{aligned}
\hat{H}_{\mathrm{eff}}= & -J \sigma_{I I}^{x}-\frac{J}{2}\left(\sigma_{I}^{x} \sigma_{I I}^{x}+\sigma_{I}^{y} \sigma_{I I}^{y}\right) \\
& +\frac{U}{4}\left(1-\hat{\sigma}_{I}^{z}\right)\left(1-\hat{\sigma}_{I I}^{z}\right) .
\end{aligned}
$$

The subscripted roman numerals are used to indicate the use of our two-qubit decomposition.

We now report quantum computations for $J=0.02$ and $U=0.03$. In the 2-qubit Hilbert space, the momentum states for $k= \pm \pi / 2$ read

$$
|k= \pm \pi / 2\rangle=\frac{1}{2}(|00\rangle \pm i|01\rangle-|10\rangle \mp i|11\rangle) .
$$

It is necessary for the initial wave packet to have some localization in space so that a distinct scattering event is visible, i.e., there is a point in time when the particle reaches the interaction region. As a side effect, the wave packet will have some momentum distribution because it no longer is a plane wave. As depicted in Fig. 5, we chose our initial wave packet to be $|\pi / 2\rangle$ but restrict it to be nonzero only in the middle:

$$
|\psi\rangle=\frac{1}{\sqrt{2}}(|01\rangle+i|10\rangle)
$$

We construct this wave packet on two sites with the following quantum circuit with all qubits initialized in the $|0\rangle$ state:

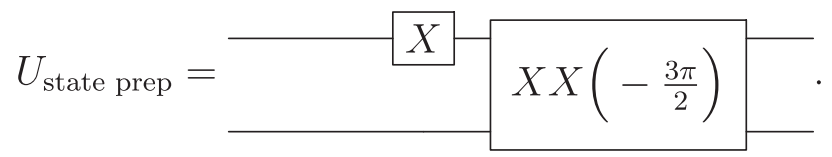

The time evolution operator can be written as a combination of $\mathrm{XX}, \mathrm{YY}, \mathrm{X}$, rotations and a controlled phase operation $\left(R_{\phi}\right)$ :

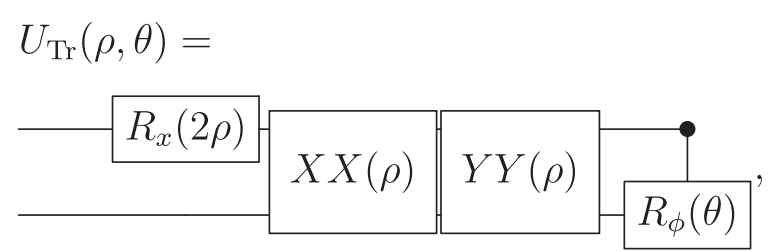

where $\rho=J \delta t, \theta=U \delta t$, and $\delta t=12.5$. We use standard notations [53] for the gates,

$$
\begin{aligned}
& X X(\rho)=e^{-i \rho X X / 2}, \quad Y Y(\rho)=e^{-i \rho Y Y / 2}, \\
& \text { and } \quad R_{X}(\rho)=e^{-i \rho X / 2} .
\end{aligned}
$$

The very slow growth of the one-step error for large $\delta t[42,43]$ allows us to reach $t=75$ with only six Trotter steps [13].

We then perform a quantum Fourier transform (QFTr) on these two qubits to take this state into momentum space:

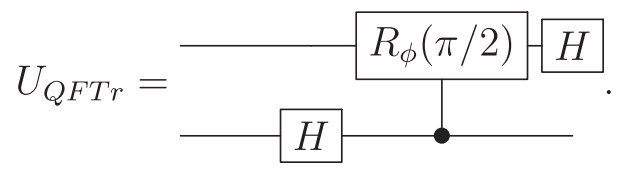

After applying the QFTr, the qubit states $|10\rangle$ and $|11\rangle$ correspond to the momentum states $|k\rangle$ and $|-k\rangle$ respectively, with $k=\pi / 2$.

The time $t^{\star}$ is determined by the symmetric condition $R_{-}\left(t^{\star}\right)=R_{+}\left(t^{\star}\right)=0.5$. Because of the small volume, we used a deformed sigmoid parametrization

$$
R_{-}(t) \simeq A /\left(1+\exp \left(-\left(\frac{t-\tilde{t}^{\star}}{w}\right)\right)\right)
$$

provides very good fits of the numerical data. The parameter $w$ describes the width of the transition region and $\tilde{t}^{\star}$ is related to $t^{\star}$ via,

$$
t^{\star}=\tilde{t}^{\star}-w \ln (2 A-1)
$$

The parameters $w$ and $\tilde{t}^{*}$ are floated during the fit. For the interacting case, there is a damping due to finite volume and coherence loss and we set $A$ equal to the last data point, which is then excluded from the fit. For the free case, the damping occurs at later time and we set $A=1$ and fit the standard sigmoid with the six data points.

The data for $R_{-}$obtained from both quantum computers are shown in Fig. 6a together with the sigmoid fits where the values of $t_{\text {free }}^{\star}$ and $t_{\text {int }}^{\star}$ are indicated by vertical lines crossing the fits at the 0.5 horizontal line. This provides the numerical values of $\Delta t^{\star}$ given in Table I. The Trotterexact and continuous-time estimates have assumed errors on par with the statistical errors from the quantum simulations, 


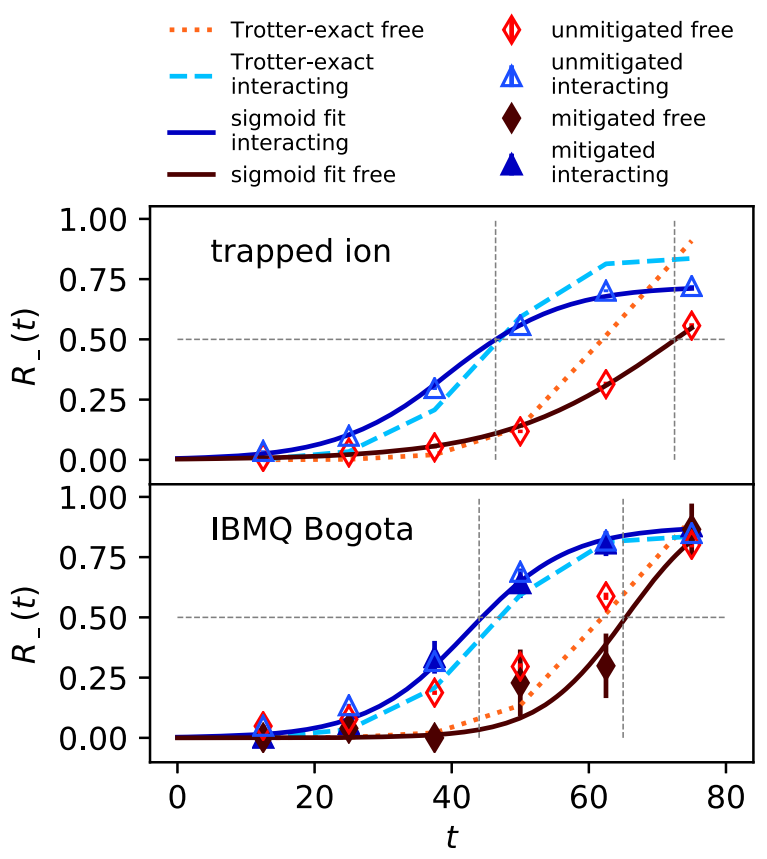

(a) Quantum mechanics limit for $R_{-}(t)$. Systematic errors shown for IBM mitigated results.

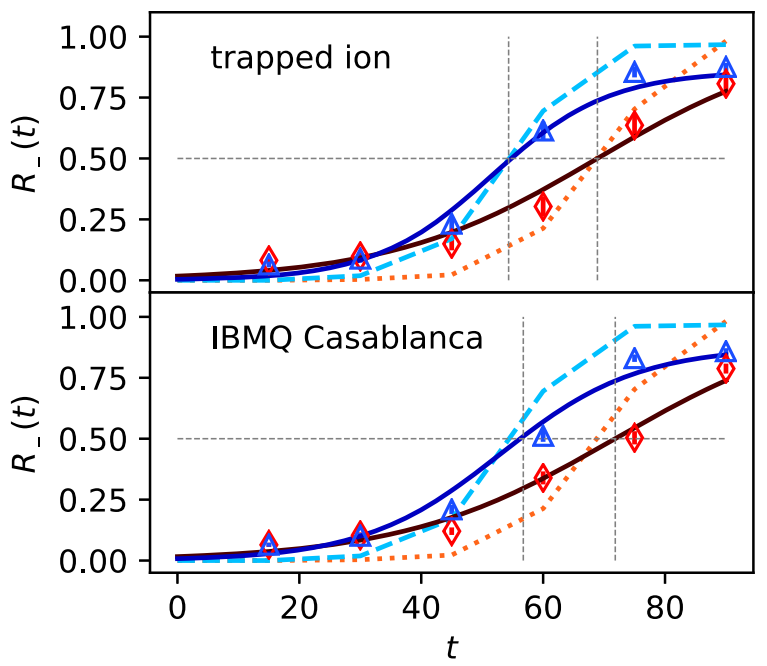

(b) Experimental results for $R_{-}(t)$ using the full Hamiltonian of Eq. (1).

FIG. 6. Experimental results for $R_{-}(t)$ using the quantum mechanics limit (a) and the full Hamiltonian of Eq. (1) (b) with and without the interaction term on four qubits for trapped ion (top in figure), IBM (bottom in figure) quantum computers. The Hamiltonians use the parameters $J=0.02, h_{T}=1.0$, and $U=0.03$. Statistical errors are shown for all except for mitigated results.

$$
\delta R_{-}(t)=\sqrt{R_{-}(t)-\left(R_{-}(t)\right)^{2}} / \sqrt{N_{\text {shots }}},
$$

TABLE I. Results for $\Delta t^{*}$ in the quantum mechanics limit (Q.M) and full field theory (Q.F.T.) from sigmoid fits of the simulated continuous and Trotter-exact evolutions as well as the experimental data from the trapped ions and IBM quantum computers.

\begin{tabular}{lcccc}
\hline \hline Type & Continuous & Trotter-exact & Trapped ions & IBM \\
\hline Q.M. & $-17.5(1)$ & $-13.7(9)$ & $-26(2)$ & $-21(2)$ \\
Q.F.T. & $-17(1)$ & $-14.3(9)$ & $-14(2)$ & $-15(2)$ \\
\hline \hline
\end{tabular}

with $N_{\text {shots }}=1000$. For comparison, we give the values obtained by doing sigmoid fits of the continuous-time evolution (first column) and the Trotter steps (second column) calculated numerically at the same discrete times as the experimental data. The readout errors for the trapped ion computer were implemented using SPAM correction. The readout errors were corrected on the IBM simulation using a pseudo-matrix inversion with least squares to ensure entirely physical values [54]. The systematic errors are expected to be larger than the statistical errors and very difficult to estimate. Details of the fits are given in the Appendix B. In addition the IBMQ Bogota simulation used Richardson extrapolations which take into account further systematic errors from the environment which produce larger uncertainties.

We see that both the IBM and trapped ion estimates provide larger absolute values of $\Delta t^{*}$ than the target values. This can be in part explained by the fact that the fits for the free process tend to lag below the Trotter steps for $t>50$ indicating a loss of coherence.

Measurements from the IBMQ Bogota machine contain both the noisy data with just readout corrections and a mitigated version obtained using methods discussed in Ref. [13] and which account for some slightly negative occupations at low $t$. This noise mitigation involves increasing the effective error rate in the circuit by applying iterated CNOT's to increase the decoherence noise and then using a linear fit to data at different noise rates to extrapolate to a noiseless limit. The trapped ion simulations include only readout corrections without noise mitigation. This is discussed in Appendix A.

We see that the quantum mechanics approximation allows us to perform the QFTr and get reasonable estimates of $\Delta t^{*}$, (Table I). We expect to improve the accuracy of these estimates in the near future. The extension of this procedure for more than four sites requires an all-to-all connectivity and a CNOT depth increasing with the number of sites. In contrast, the field theory calculation discussed in the next section, and which is our ultimate goal, requires more qubits but remains local [52] with a constant CNOT depth. 


\section{TOWARD SCALABLE FIELD THEORY CALCULATIONS}

We have performed field theory computations with the same devices using four sites and four qubits. This allows for shallower local circuits but requires a more expensive Fourier transform [55]. The initial state that we prepared,

$$
\left|\psi_{i}\right\rangle=\frac{1}{\sqrt{2}}(|01\rangle+i|10\rangle)
$$

can be remapped to the Ising model by using the inverse mapping originally described in the text:

$$
\begin{aligned}
|00\rangle & \rightarrow|1000\rangle, & |01\rangle & \rightarrow|0100\rangle \\
|10\rangle & \rightarrow|0010\rangle, & |11\rangle & \rightarrow|0001\rangle .
\end{aligned}
$$

This remapping will give us the initial state:

$$
\left|\psi_{i}\right\rangle=\frac{1}{\sqrt{2}}(|0100\rangle+i|0010\rangle) .
$$

The time evolution of the system can be easily written in terms of a circuit requiring at most $3 X X$ gates per Trotter step when using a four site system, which is $2 X X$ gates deep, see Eq. (33).

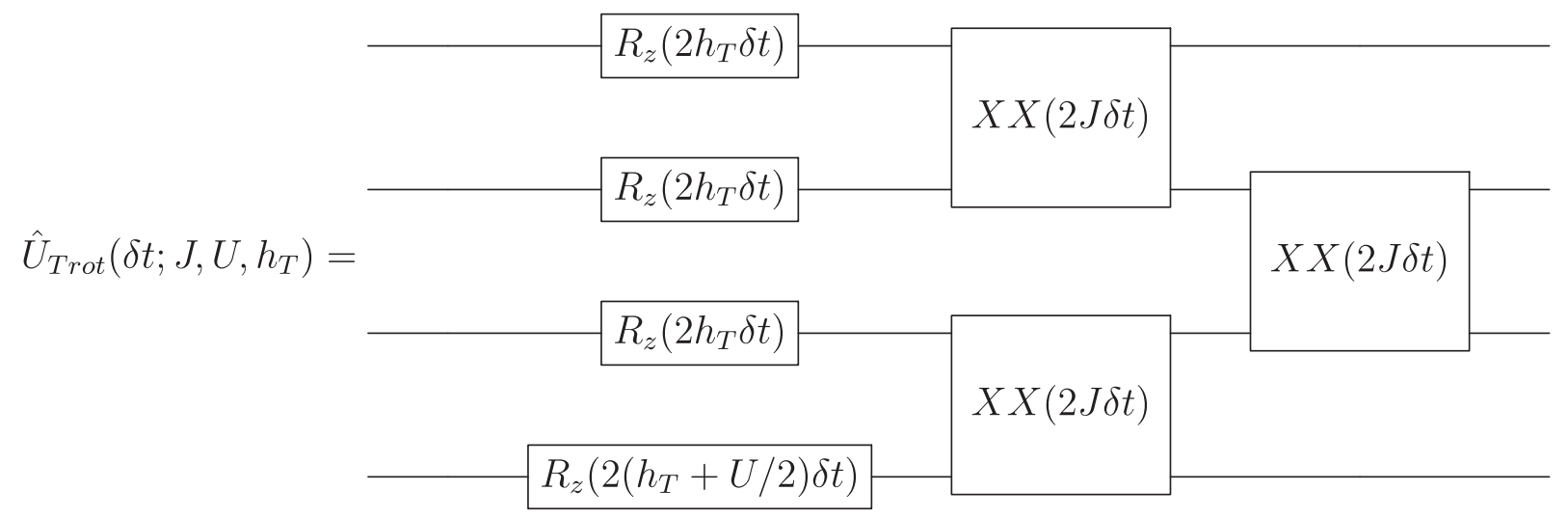

The final portion of the quantum simulation is a Fourier transformation to take the circuit into momentum space [56,57]. This transformation can easily be done using four two-qubit operations.

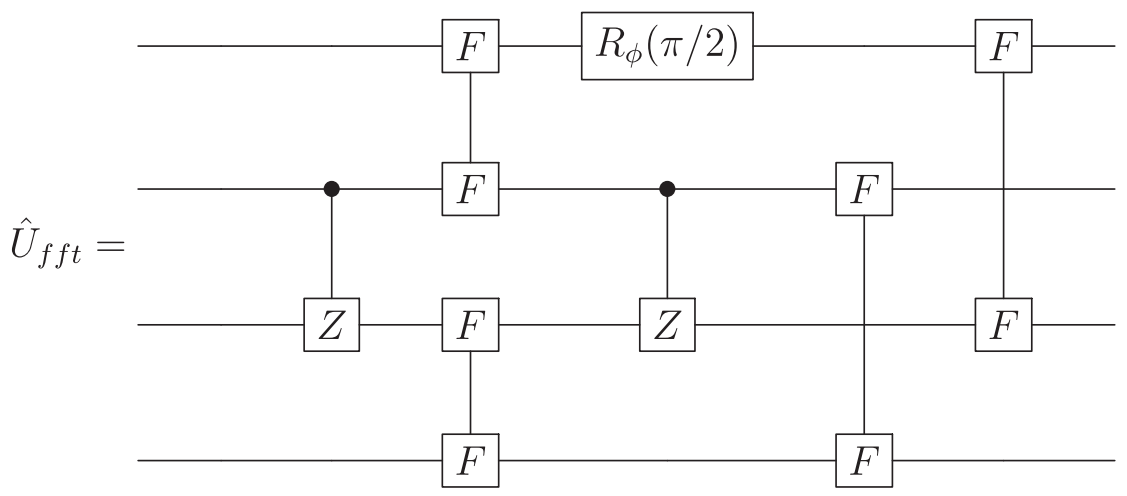


Where $F$ is given by the matrix:

$$
F=\left(\begin{array}{cccc}
1 & 0 & 0 & 0 \\
0 & \frac{1}{\sqrt{2}} & \frac{1}{\sqrt{2}} & 0 \\
0 & \frac{1}{\sqrt{2}} & -\frac{1}{\sqrt{2}} & 0 \\
0 & 0 & 0 & -1
\end{array}\right) .
$$

This can be decomposed into at most 4 CNOT gates. It should be noted this is different from the traditional quantum Fourier transform which uses binary encoding for the qubits. The decompositions for the $F$-gate, $C Z$, and $X X$ are shown in Appendix D.

The measurement is done then in the z-basis and we select out the states $|0010\rangle$ and $|0001\rangle$ for the $|k\rangle$ and $|-k\rangle$ state respectively.

In Fig. 6(b) we show the results of these calculations on IBM's Casablanca and the Maryland ion trap machines using the full Hamiltonian with a Trotter step of $\delta t=15$ with six Trotter steps. In both cases, unmitigated data is used for the analysis. Figure 6(b) indicates that the discrepancies between the individual fits and the Trotter exact results are more pronounced than in the previous calculation. However the effects appear to somehow compensate when we calculate the differences and we obtain time delays closer to the Trotter exact ones as shown in Table I. This question could be investigated with more accurate data. As discussed in the SM, the fit methods are similar to the previous ones. No Richardson extrapolations are performed in this simulation because the effective noise rate on the circuit would be well outside the linear regime for the error. The nonlinear errors would render a Richardson extrapolation infeasible. As improved quantum computing hardware platforms become available, we plan to use these upgraded facilities to get more accurate values of $\Delta t^{\star}$ and extend these calculations to 8 and 16 sites with one qubit per site. We provide the gate costs for each of these simulations in Tables IV and V in Appendix C. The circuit cost of the of the Fourier transform is expected to be the most expensive but still scales like $\mathcal{O}(n \log (n))$ [55].

\section{CONCLUSIONS}

We have proposed a method to estimate the time delay using the early steps of the real-time evolution. We have given a proof of principle that actual computations of the time delays can be implemented on both the IBM superconducting transmon and trapped ion hardware platforms for a quantum mechanics limit with two qubits and the field theory formulation with four qubits. There is plenty of room for optimization for both devices and we do not claim that our results allow a systematic comparison between the devices. We expect that the field theory calculations should feasible for a larger number of qubits in the near future. A detailed comparison with existing real-time methods in one spatial dimension [58-61] would be of great interest. Quantum computations for quantum Ising models in two spatial dimensions could offer the possibility to reach quantum advantage.

\section{ACKNOWLEDGMENTS}

E. Gustafson and Y. Meurice were supported in part by the U.S. Department of Energy (DoE) under Award No. DE-SC0019139. They thank the members of QuLAT for suggestions and comments. They also thank Wayne Polyzou and the math physics seminar at the University of Iowa for fruitful discussions. P. Dreher was supported in part by the U.S. Department of Energy (DoE) under Award No. DE-AC05-00OR22725. We thank North Carolina State University (NCSU) for access to the IBM Q Network quantum computing hardware platforms through the NCSU IBM Q Hub. N. M. Linke acknowledges support by the DoE NP Quantum Horizons program, Award No. DESC0021143, and the NSF Grant No. PHY-1430094 to the PFC@JQI. We are extremely grateful to Alaina M. Green, Cinthia Huerta Alderete and Nhung H. Nguyen for help with the trapped-ion measurements.

\section{APPENDIX A: QUANTUM COMPUTING HARDWARE PLATFORMS}

This project used both the IBM Quantum Network superconducting transmon hardware platforms and a trapped ion machine. For the IBM quantum computers, the project used $\mathrm{IBM}_{Q}$ Bogota (5 qubit) and $\mathrm{IBM}_{Q}$ Casablanca (7 qubit) machines. Both IBM platforms are rated with a quantum volume of 32 . Access to these machines was through the IBM cloud via the NC State IBM Quantum Hub.

The ion trap quantum computer used in this study consists of a chain of ${ }^{171} \mathrm{Yb}^{+}$ions confined in a linear Paul trap and laser-cooled to their motional ground states [51,62]. The qubits are defined in the hyperfine-split ${ }^{2} S_{1 / 2}$ manifold as $|0\rangle=\left|F=0, m_{F}=0\right\rangle,|1\rangle=\left|F=1, m_{F}=0\right\rangle$, with a splitting of $12.642821 \mathrm{GHz}$ and are insensitive to magnetic field fluctuations to first order. Qubits are initialized to the $|0\rangle$ state by optical pumping [63]. Coherent operations are achieved by illuminating the ions with a pair of counterpropagating Raman beams at 355-nm which have a beat-note that is set resonant with the qubits or near the motional sidebands of the chain [64]. One of the Raman beams illuminates the entire chain. The other one is split into individual beams that are each matched onto one channel on a multi-channel acoustic-optic modulator (AOM). The amplitude, frequency, and phase of each beam can be modulated independently by the corresponding $\mathrm{rf}$ signal. Individual addressing is achieved by focusing each of these beams onto one single ion. Each ion is also matched onto a distinct channel of a photomultiplier tube 
(PMT) that collects state-dependent fluorescence for individual state readout [63]. There are two mechanisms of quantum control. Single qubit rotations are performed by driving Rabi rotations of the target ion on resonance. Twoqubit entanglement is implemented by creating an effective Ising spin-to-spin interaction via transient entanglement between the qubits and the motional modes with the Raman beat-note tuned near the motional sidebands [65-67]. Only qubit-to-qubit entanglement remains after the motional modes are disentangled at the end of the scheme [68]. Entanglement can be created between any pair of ions owing to the long-range Coulomb interaction [69]. Typical gate fidelities are around 99.5(2)\% for the single-qubit gates and 98(1)\% for the two-qubit entangling gates. The main sources of two-qubit gate errors are residual entanglement between the motional modes and the qubits due to laser intensity fluctuations and motional heating. State preparation and measurement (SPAM) errors are accounted for by applying the inverse of an independently measured state-to-state error matrix when analyzing the data.

\section{APPENDIX B: DETERMINING TIME DELAY WITH SIGMOIDS}

We find the time delay using the sigmoid for the fit of the quantities $R_{-}(t)$. The results for the quantum mechanics simulations are shown in Table II and for the Quantum Field theory simulations are shown in Table III. We have

TABLE II. Result of sigmoid fits to a sampling of the exact evolution operator (continuous), Trotterization, the unmitigated and mitigated data from IBM hardware, and the University of Maryland trapped ion quantum computer (quantum mechanics limit).

\begin{tabular}{lccccc}
\hline \hline Type & $\tilde{t}_{\text {free }}^{\star}$ & $\tilde{t}_{\text {int }}^{\star}$ & $\Delta t^{\star}$ & $w_{\text {free }}$ & $w_{\text {int }}$ \\
\hline Continuous Sampled & $68.1(1)$ & $50.6(2)$ & $-17.5(1)$ & $6.47(8)$ & $7.12(2)$ \\
Trotterization & $61.8(6)$ & $48.2(7)$ & $-13.7(9)$ & $5.8(4)$ & $8.8(6)$ \\
IBM unmitigated & $57.9(8)$ & $40(1)$ & $-13(1)$ & $13.6(5)$ & $8.9(2)$ \\
IBM mitigated & $65(3)$ & $47(1)$ & $-21(3)$ & $6(2)$ & $10.4(7)$ \\
Trapped ion & $72.6(6)$ & $39.6(2)$ & $-26(2)$ & $12.5(4)$ & $8.2(2)$ \\
\hline \hline
\end{tabular}

TABLE III. Result of sigmoid fits to a sampling of the exact evolution operator (continuous), Trotterization, the unmitigated from IBM hardware, and the University of Maryland Trapped Ion quantum computer (QFT).

\begin{tabular}{lccccc}
\hline \hline Type & $\tilde{t}_{\text {free }}^{\star}$ & $\tilde{t}_{\text {int }}^{\star}$ & $\Delta t^{\star}$ & $w_{\text {free }}$ & $w_{\text {int }}$ \\
\hline Continuous Sampled & $68.0(7)$ & $50.9(8)$ & $-17(1)$ & $5.25(3)$ & $8.2(2)$ \\
Trotterization & $69.1(6)$ & $54.8(7)$ & $-14.3(9)$ & $6.8(6)$ & $6.5(4)$ \\
IBM unmitigated & $72(2)$ & $57(2)$ & $-15(2)$ & $18(1)$ & $11(1)$ \\
Trapped ions & $69(1)$ & $54(1)$ & $-14(2)$ & $17(1)$ & $9.6(8)$ \\
\hline \hline
\end{tabular}

separated out each of the various data sets into lines. The continuous sampling uses 100 equally spaced time steps between $t=0$ and $t=75$ with a corresponding uncertainty of 0.001 . Similarly, we also used 6 equally spaced data points sampled from the continuous distribution at times which are multiples of $t=12.5$ with the same uniform error rate of 0.001 . The noiseless Trotter (Trotterexact) was sampled at times steps of $\delta t=12.5$ and had an uncertainty of $\delta P_{ \pm}(t)=\sqrt{P_{ \pm}-P_{ \pm}^{2}(t)} / \sqrt{1000}$. The remaining three lines were sampled by fitting to the first five data points for the interacting case (int) and six data points for the free case. This was done to make sure that the value of $R_{-}(t)$ is at least 0.5 and that the system has not reached an asymptotic state. Stated in a different way, in the interacting case, the signal flattens below 1 after 5 Trotter steps due to the reflection on the left wall.

It is possible to get an idea of the magnitude of the systematic errors by looking at the first time step, where a detailed examination shows that the measured values of $R_{-}$ are significantly larger than the Trotter exact values and the estimated statistical errors. In absence of a reliable model for the systematic errors, we estimated the error on the parameters using the "jackknife" method, which involves iteratively removing one data point and fitting to this smaller data set and then using the standard deviation to measure the systematic uncertainty.

The fitting methods for the field theory results are similar, the only difference being a slightly larger Trotter step.

\section{APPENDIX C: GATE COSTS}

TABLE IV. Total gate costs for IBM using native gates $U(\theta, \lambda, \phi)$ and CNOT.

\begin{tabular}{lcccccc}
\hline \hline Operation & & \multicolumn{2}{c}{ Quantum } & \multirow{2}{*}{ Typechanics } & & \multicolumn{2}{c}{ Field theory } \\
\cline { 3 - 4 } \cline { 6 - 7 } \cline { 6 - 7 } & & 1 qubit & 2 qubit & & 1 qubit & 2 qubit \\
\hline State preparation & 2 & 2 & & 2 & 2 \\
Single Trotter & 15 & 3 & & 7 & 6 \\
Fourier transform & 5 & 2 & & 44 & 14 \\
\hline \hline
\end{tabular}

TABLE V. Total gate costs for trapped ions using native gates $X X(\phi)$ and native 1-qubit rotations.

\begin{tabular}{|c|c|c|c|c|}
\hline \multirow{2}{*}{$\begin{array}{l}\text { Operation } \\
\text { Type }\end{array}$} & \multicolumn{2}{|c|}{ Quantum mechanics } & \multicolumn{2}{|c|}{ Field theory } \\
\hline & 1 qubit & 2 qubit & 1 qubit & 2 qubit \\
\hline State preparation & 2 & 2 & 2 & 2 \\
\hline Single trotter & 8 & 3 & 4 & 3 \\
\hline Fourier transform & 5 & 1 & 52 & 14 \\
\hline
\end{tabular}




\section{APPENDIX D: GATE DECOMPOSITIONS}

In this section we provide the gate decompositions for the $F$-gate and the $C Z$ gate. For the Maryland trapped ion quantum computer the $C Z$ gate is given in Fig. 7 and the $F$-gate is given in Fig. 8. The implementation of the $F$-gate on the IBM quantum hardware is provided in Fig. 9.

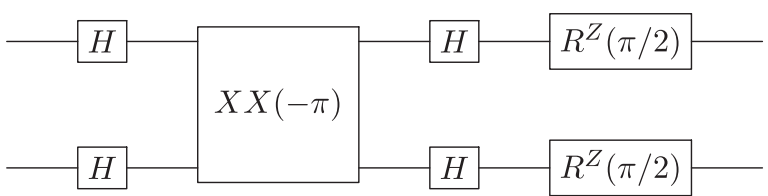

FIG. 7. Decomposition of $C Z$ for Maryland trapped ion quantum computer.

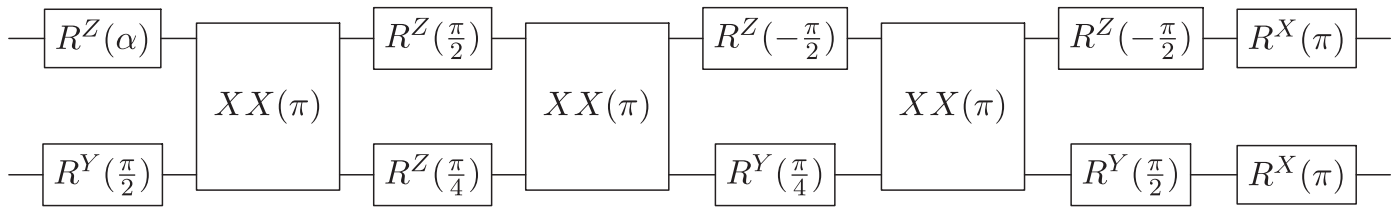

FIG. 8. Decomposition of F-gate for Maryland trapped ion quantum computer.

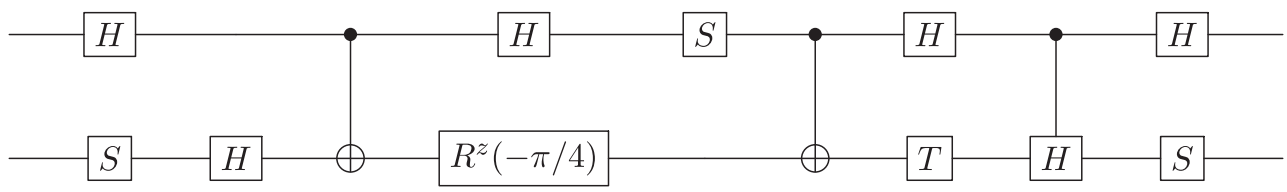

FIG. 9. Decompostion of F-gate for IBM quantum computer.

[1] J. Preskill, in Proceedings of Science, Lattice Conference 2018 (Michigan State University, Michigan, 2018), https:// pos.sissa.it/334/024/.

[2] See review 17 of the PDG [70].

[3] T. Sjöstrand, S. Mrenna, and P. Skands, J. High Energy Phys. 06 (2006) 026.

[4] M. Bähr, S. Gieseke, M. Gigg, D. Grellscheid, K. Hamilton, S. Latunde-Dada, S. Plätzer, P. Richardson, M. Seymour, A. Sherstnev, and B. Webber, Eur. Phys. J. C 58, 639 (2008).

[5] N. Mueller, A. Tarasov, and R. Venugopalan, Phys. Rev. D 102, 016007 (2020).

[6] H. Lamm, S. Lawrence, and Y. Yamauchi, Phys. Rev. Research 2, 013272 (2020).

[7] J. Preskill, Quantum information and space-time (2017), http://theory.caltech.edu/ preskill/talks/QIP2017-tutorialPreskill.pdf.

[8] J. B. Kogut, Rev. Mod. Phys. 51, 659 (1979).

[9] J. B. Kogut, Rev. Mod. Phys. 55, 775 (1983).

[10] A. Cervera-Lierta, Quantum 2, 114 (2018).

[11] H. Lamm and S. Lawrence, Phys. Rev. Lett. 121, 170501 (2018).

[12] E. Gustafson, Y. Meurice, and J. Unmuth-Yockey, Phys. Rev. D 99, 094503 (2019).
[13] E. Gustafson, P. Dreher, Z. Hang, and Y. Meurice, Quantum Sci.Technol. 6, 4 (2021).

[14] M. Kim, Y. Song, J. Kim, and J. Ahn, PRX Quantum 1, 020323 (2020).

[15] K. Yeter-Aydeniz, G. Siopsis, and R. C. Pooser, New J. Phys. 23, 043033 (2021).

[16] J. Vovrosh and J. Knolle, Sci. Rep. 11, 11577 (2021).

[17] Y. Salathé, M. Mondal, M. Oppliger, J. Heinsoo, P. Kurpiers, A. Potočnik, A. Mezzacapo, U. Las Heras, L. Lamata, E. Solano et al., Phys. Rev. X 5, 021027 (2015).

[18] W. L. Tan, P. Becker, F. Liu, G. Pagano, K. S. Collins, A. De, L. Feng, H. B. Kaplan, A. Kyprianidis, R. Lundgren et al., Nat. Phys. 17, 742 (2021).

[19] A. Smith, M. S. Kim, F. Pollmann, and J. Knolle, Quantum Inf. 5, 106 (2019).

[20] H. Labuhn, D. Barredo, S. Ravets, S. de Léséleuc, T. Macrì, T. Lahaye, and A. Browaeys, Nature (London) 534, 667 (2016).

[21] J. Zhang, G. Pagano, P. W. Hess, A. Kyprianidis, P. Becker, H. Kaplan, A. V. Gorshkov, Z. X. Gong, and C. Monroe, Nature (London) 551, 601 (2017).

[22] T. Kadowaki and H. Nishimori, Phys. Rev. E 58, 5355 (1998). 
[23] H. Bernien, S. Schwartz, A. Keesling, H. Levine, A. Omran, H. Pichler, S. Choi, A. S. Zibrov, M. Endres, M. Greiner, V. Vuletić, and M. D. Lukin, Nature (London) 551, 579 (2017).

[24] A. Alexandru, P. F. Bedaque, S. Harmalkar, H. Lamm, S. Lawrence, and N. C. Warrington, Phys. Rev. D 100, 114501 (2019).

[25] E. Zohar, J. I. Cirac, and B. Reznik, Phys. Rev. A 88, 023617 (2013).

[26] E. A. Martinez, C. A. Muschik, P. Schindler, D. Nigg, A. Erhard, M. Heyl, P. Hauke, M. Dalmonte, T. Monz, P. Zoller et al., Nature (London) 534, 516 (2016).

[27] B. Buyens, J. Haegeman, F. Hebenstreit, F. Verstraete, and K. Van Acoleyen, Phys. Rev. D 96, 114501 (2017).

[28] N. Klco, E. F. Dumitrescu, A. J. McCaskey, T. D. Morris, R. C. Pooser, M. Sanz, E. Solano, P. Lougovski, and M. J. Savage, Phys. Rev. A 98, 032331 (2018).

[29] R. C. Brower, D. Berenstein, and H. Kawai, Proc. Sci., LATTICE2019 (2020) 112.

[30] P. I. Karpov, G. Y. Zhu, M. P. Heller, and M. Heyl, arXiv:2011.11624.

[31] E. T. Holland, K. A. Wendt, K. Kravvaris, X. Wu, W. E. Ormand, J. L. DuBois, S. Quaglioni, and F. Pederiva, Phys. Rev. A 101, 062307 (2020).

[32] A. Roggero, C. Gu, A. Baroni, and T. Papenbrock, Phys. Rev. C 102, 064624 (2020).

[33] F. M. Surace and A. Lerose, New J. Phys. 23, 062001 (2021).

[34] D. E. Kharzeev and Y. Kikuchi, Phys. Rev. Research 2, 023342 (2020).

[35] K. Ikeda, D. E. Kharzeev, and Y. Kikuchi, Phys. Rev. D 103, L071502 (2021).

[36] R. A. Briceño, J. V. Guerrero, M. T. Hansen, and A. Sturzu, Phys. Rev. D 103, 014506 (2021).

[37] M. Van Damme, L. Vanderstraeten, J. De Nardis, J. Haegeman, and F. Verstraete, Phys. Rev. Research 3, 013078 (2021).

[38] M. C. Bañuls et al., Eur. Phys. J. D 74, 165 (2020).

[39] L. F. Richardson and J. A. Gaunt, Phil. Trans. R. Soc. A 226, 299 (1927).

[40] A. Kandala, K. Temme, A. D. Córcoles, A. Mezzacapo, J. M. Chow, and J. M. Gambetta, Nature (London) 567, 491 (2019).

[41] A. Kandala, A. Mezzacapo, K. Temme, M. Takita, M. Brink, J. M. Chow, and J. M. Gambetta, Nature (London) 549, 242 (2017).

[42] Y. Meurice, Quantum Field Theory: A Quantum Computation Approach (Institute of Physics Publishing, Bristol, 2021).

[43] Y. Meurice, R. Sakai, and J. Unmuth-Yockey, arXiv:2010.06539.

[44] E. P. Wigner, Phys. Rev. 98, 145 (1955).

[45] M. Lüscher, Commun. Math. Phys. 105, 153 (1986).

[46] R. A. Briceño, Z. Davoudi, T. Luu, and M. J. Savage, Phys. Rev. D 88, 114507 (2013).

[47] R. A. Briceno, J. J. Dudek, R. G. Edwards, and D. J. Wilson, Phys. Rev. Lett. 118, 022002 (2017).

[48] R. A. Briceno, J. J. Dudek, and R. D. Young, Rev. Mod. Phys. 90, 025001 (2018).
[49] M. J. Savage, in Proceedings, 34th International Symposium on Lattice Field Theory (Lattice 2016): Southampton, UK, 2016; Proc. Sci., LATTICE2016 (2016) 021 [arXiv:1611.02078].

[50] Z. Davoudi, Proc. Sci., LATTICE2018 (2018) 014 [arXiv:1812.11899].

[51] S. Debnath, N. M. Linke, C. Figgatt, K. A. Landsman, K. Wright, and C. Monroe, Nature (London) 536, 63 (2016).

[52] S. Lloyd, Science 273, 1073 (1996).

[53] M. Nielsen and I. Chuang, Quantum Computation and Quantum Information, Cambridge Series on Information and the Natural Sciences (Cambridge University Press, Cambridge, England, 2000).

[54] I. Research and the IBM QX team, IBM Q Experience User Guide, https://qiskit.github.io/ibmqx-user-guides/full-userguide/introduction.html (2017).

[55] A. J. Ferris, Phys. Rev. Lett. 113, 010401 (2014).

[56] A. J. Ferris, Phys. Rev. Lett. 113, 010401 (2014).

[57] I. D. Kivlichan, C. Gidney, D. W. Berry, N. Wiebe, J. McClean, W. Sun, Z. Jiang, N. Rubin, A. Fowler, A. Aspuru-Guzik et al., Quantum 4, 296 (2020).

[58] G. Vidal, Phys. Rev. Lett. 93, 040502 (2004).

[59] S. R. White and A. E. Feiguin, Phys. Rev. Lett. 93, 076401 (2004).

[60] F. Verstraete, J. J. García-Ripoll, and J. I. Cirac, Phys. Rev. Lett. 93, 207204 (2004).

[61] A. J. Daley, C. Kollath, U. Schollwöck, and G. Vidal, J. Stat. Mech. (2004) P04005.

[62] K. A. Landsman, C. Figgatt, T. Schuster, N. M. Linke, B. Yoshida, N. Y. Yao, and C. Monroe, Nature (London) 567, 61 (2019).

[63] S. Olmschenk, K. C. Younge, D. L. Moehring, D. N. Matsukevich, P. Maunz, and C. Monroe, Phys. Rev. A 76, 052314 (2007).

[64] R. Islam, W. C. Campbell, T. Choi, S. M. Clark, C. W. S. Conover, S. Debnath, E. E. Edwards, B. Fields, D. Hayes, D. Hucul, I. V. Inlek, K. G. Johnson, S. Korenblit, A. Lee, K. W. Lee, T. A. Manning, D. N. Matsukevich, J. Mizrahi, Q. Quraishi, C. Senko, J. Smith, and C. Monroe, Opt. Lett. 39, 3238 (2014).

[65] G. J. Milburn, S. Schneider, and D. F. V. James, Fortschr. Phys. 48, 801 (2000).

[66] K. Mølmer and A. Sørensen, Phys. Rev. Lett. 82, 1835 (1999).

[67] E. Solano, R. L. de Matos Filho, and N. Zagury, Phys. Rev. A 59, R2539 (1999).

[68] T. Choi, S. Debnath, T. Manning, C. Figgatt, Z.-X. Gong, L.-M. Duan, and C. Monroe, Phys. Rev. Lett. 112, 190502 (2014).

[69] N. M. Linke, D. Maslov, M. Roetteler, S. Debnath, C. Figgatt, K. A. Landsman, K. Wright, and C. Monroe, Proc. Natl. Acad. Sci. U.S.A. 114, 3305 (2017).

[70] P. D. Group, P. A. Zyla et al., Prog. Theor. Exp. Phys. 2020, 083C01 (2020). 